\title{
Deciphering clinical phenotypes in acute viral lower respiratory tract infection: Bronchiolitis is not an island
}

\author{
Steve Cunningham, ${ }^{1}$ Harish Nair, ${ }^{2}$ Harry Campbell ${ }^{2}$
}

Bronchiolitis is an internationally recognised diagnosis that is commonly applied to children across healthcare systems. In the UK, $2 \%$ of all infants are admitted to paediatric hospitals each year with a diagnosis of bronchiolitis, typically over 6-8 weeks winter peak period. Globally, bronchiolitis is just as common though seasons are more diffuse and variable in tropical climates. For such a common disease, there remain significant gaps in our understanding of its pathophysiology and also its interrelationship with other common lower respiratory tract disease in young children. This lack of data is now restraining progress in the development of management and therapeutics for the preschool age group who are most affected by lower respiratory tract disease (both for infections, ie, respiratory syncytial virus (RSV) and for the response to infection, ie, wheeze phenotypes). So the study by Dumas et al in Thorax is welcome, as they attempt to stratify subgroups of patients with a clinical diagnosis of bronchiolitis. ${ }^{1}$

Yet, despite being a common condition in young children, it has proven challenging to develop an international consensus on what precisely defines bronchiolitis from a clinical perspective and how this differs from other common lower respiratory conditions. Most paediatricians consider that they would instantly recognise a case, but agreement may be less consistent when clinicians are less experienced, children are older, have clinical risk factors or present outside the peak RSV season. In many ways this reflects gaps in our current knowledge about lower respiratory tract disease in young children, as the diagnosis of bronchiolitis is intended to be the clinical representation of typical histological features within the bronchioles of those affected: oedematous bronchioles filled with necrotic epithelium and neutrophils, bound together by mucous. ${ }^{2}$ As a

\footnotetext{
${ }^{1}$ Department of Child Life and Health, University of Edinburgh, Edinburgh, UK; ${ }^{2}$ Centre for Global Health Research, University of Edinburgh, Edinburgh, UK

Correspondence to Prof Steve Cunningham, Department of Child Life and Health, University of Edinburgh, Sylvan Place, Edinburgh, EH9 1LF, UK; steve.cunningham@nhs.net
}

histological review of only the most severe cases of bronchiolitis (resulting in death) have informed this understanding, we do not have a comprehensive overview of the range of pathologies associated with the wider spectrum of clinical symptoms and age ranges where a clinical diagnosis of bronchiolitis may be given.

In the study by Dumas et al, children were included if they were given a diagnosis of bronchiolitis by a physician in a secondary care setting in the USA and Finland. This large study used a clustering methodology novel to bronchiolitis research. However, the diagnosis of bronchiolitis is considered inconsistent across and within healthcare settings and, despite the use of study protocols, this may have influenced the ability of the study to adequately describe subgroups in order to assess the generalisability of the findings to other settings. We cannot be sure that a child with the same signs and symptoms arriving at an emergency department being seen by a different doctor or in different study centres would be labelled as bronchiolitis as opposed to another diagnosis consistent with a lower respiratory tract infection, leading to incomplete or inconsistent acquisition of cases.

Internationally, bronchiolitis guidelines have struggled for many years to configure a set of clinical symptoms and signs to a specific unifying diagnosis of bronchiolitis, with a low probability for alternative diagnoses. Five guidelines have been published in English since 2010 with each providing clinical features considered to define bronchiolitis (South Africa, ${ }^{3}$ Canada, ${ }^{4}$ USA, ${ }^{5}$ UK $^{6}$ and Finland ${ }^{7}$ ). These show significant variation for example, in terms of the targeted age group and specific symptoms and clinical signs:

- Age: In the USA and Canada, guidelines limit the diagnosis to 0-2 years of age, while the South African and UK guidelines recognise the predominance in infants and note the potential for cases across preschool age ranges, especially in those considered at high risk, and so make no limit on age. In the Finnish guideline, bronchiolitis is considered predominantly under 6 months of age.

- Symptoms: All guidelines recognise coryza and cough as phenotypical of bronchiolitis, but these symptoms occur up to 10 times per year in young children and so poorly differentiate bronchiolitis from other respiratory infections.

- Clinical signs: All guidelines consider signs of lower respiratory tract infection (tachypnoea, chest retractions and wheezing) to be important; crackles on auscultation is also noted as an optional finding in guidelines from Canada, UK and Finland but these clinical signs are also common to other clinical phenotypes in this age range-virus-induced wheeze and viral pneumonia. In the Finnish guideline, the presence of wheeze in those aged over 1 year is considered to denote a diagnosis of 'wheezy bronchitis', particularly when associated with rhinovirus (whereas, in the UK, 'viral induced wheeze' and in the USA, 'bronchiolitis' would be more commonly used diagnostic terms used at this age).

But it is not just between nations where discrepancies lie-across the UK, there is significant variation-both in primary and secondary care settings. In the UK, an analysis of primary care databases reported that a specific diagnosis of bronchiolitis was given in just 58-65 per 1000 children, but that this number increased threefold to 204 per 1000 when a broader definition was used to reflect lower respiratory tract infection consistent with a diagnosis of bronchiolitis. $^{8} 9$ Similar, but smaller, studies from Spain and Bangladesh also suggest that the diagnostic term of 'bronchiolitis' in children with viral lower respiratory tract infection is more common in secondary care settings where there is paediatric experience. ${ }^{10}{ }^{11} \mathrm{In}$ the UK, the rates of secondary care admissions coded as bronchiolitis vary up to fivefold across hospitals in different health regions. ${ }^{12}$ With pressure on paediatric inpatient services, admissions to hospital with bronchiolitis are typically reserved for those who are hypoxaemic or have inadequate feeding and so it is likely that much of this variation in admission rates represents differentially coded diagnoses for the same clinical symptoms across UK hospitals.

So imprecise are the definitions for bronchiolitis with so much overlap of clinical features with related common clinical conditions in the preschool years that children with the same clinical condition seem likely to receive different diagnostic labels depending on factors associated with the doctor, hospital and country where the children are assessed. If diagnostic labelling of bronchiolitis can be so inconsistent, then is the diagnostic term of 'bronchiolitis' helpful or, as we are unclear about the range of pathophysiology across phenotypes, should we assign these symptoms in 
purely descriptive term such as "viral acute lower respiratory tract infection'?

Before the advent of PCR virus diagnostics, there was a perceived advantage to provide a diagnosis of bronchiolitis, as it inferred viral origin. The range of viruses that can now be identified by PCR testing with very high detection rates has now enabled much greater confidence in the diagnosis of a viral aetiology of lower respiratory tract infections, where clinical signs can be similar to those of bacterial pneumonia. ${ }^{13}$ With this, a clinical label of bronchiolitis is possibly less necessary than previously when such viral diagnostics were not available or not so widely applied. With increasing concern about antimicrobial resistance, as a major public health concern, the application of the term 'viral lower respiratory tract infection' may help encourage restriction of the use of antibiotics to only those who have additional clinical features (eg, persisting fever and/or focal crepitations) consistent with bacterial superadded infection. Viral diagnostics are enhancing our understanding of the role of virus infections across symptom profiles and different age ranges, in particular in relation to viral co-infections. ${ }^{14}$

Acute viral lower respiratory tract infection in young children results in general symptoms of viral respiratory tract infection (coryza and cough) and lower respiratory tract signs (increased work of breathing, fast breathing, wheeze, crackles) in association with an identified viral infection. In many circumstances acute viral lower respiratory tract infection is already used as the main diagnostic term. At a global level, the most commonly used clinical guidelines are for first-line health workers and contained in the WHO Integrated Management of Childhood Illness (IMCI) approach (http://www.who. int/maternal_child_adolescent/documents/ IMCI_chartbooklet/en/). For children presenting with respiratory symptoms, IMCI trains health workers to identify young children with clinical 'pneumonia' who require antibiotic treatment at home or hospital admission for antibiotic treatment, oxygen therapy and supportive care. Clinical pneumonia is a mixed bag of clinical conditions. The IMCI guidelines are targeted at first-level health workers and, therefore, do not attempt to distinguish between pneumonia and bronchiolitis. This is in part due to problems of poor discrimination noted above and in part due to the low levels of understanding of the term 'bronchiolitis' by most child health workers globally. For estimates of disease burden, ${ }^{15}$ 'viral lower respiratory tract infection' has been a more effective term to capture disease in young children (since the term 'bronchiolitis' is not widely used in low-income and middle-income country studies). In the development of new therapeutics for RSV, various protocols (including candidate case definitions developed by WHO for RSV vaccine trials) have not required a diagnosis of 'bronchiolitis' and have rather used the term 'lower respiratory tract infection with proven RSV infection'. ${ }^{16} 17$ This has removed the requirement for clinicians to independently gauge whether common symptoms fit within the constrained diagnosis of bronchiolitis and tests whether treatment of the infecting agent will affect disease course regardless of the diagnostic label. We consider that such evaluations are appropriate and will better benefit patients than restricting the intervention and licence to a limited clinical diagnosis.

With fewer effective management and treatment options for lower respiratory tract symptoms in preschool children than in any other age group, there is an urgent need to build a strong evidence base on which to base future recommendations. This lack of evidence is apparent in the difficulties encountered by clinical guidelines, that struggle to be inclusive for children over 6 months of age with 'bronchiolitis' and equally for children below 5 years of age with 'asthma'. Pigeonholing diagnoses in this age group as square pegs into round holes is not helping in elucidating and evolving our understanding of the pathophysiology of disease and in identifying effective biomarkers of lower respiratory tract infection for those most commonly affected-children under 5 years of age. For a true evaluation, larger studies that are unconstrained by current diagnostic labelling are warranted.

Contributors SC drafted the first draft of the manuscript that was then developed and finalised by all three authors.

\section{Competing interests None declared.}

Provenance and peer review Commissioned; externally peer reviewed.

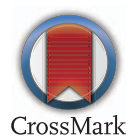

To cite Cunningham S, Nair H, Campbell H. Thorax 2016;71:679-680.

Published Online First 7 July 2016

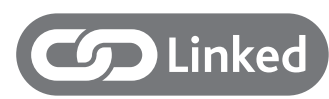

http://dx.doi.org/10.1136/thoraxjnl-2016-208535
Thorax 2016;71:679-680.

doi:10.1136/thoraxjnl-2016-209012

\section{REFERENCES}

1 Dumas 0, Mansbach JM, Jartti T, et al. A clustering approach to identify severe bronchiolitis profiles in children. Thorax 2016;71:712-8.

2 Zinserling A. Pecularities of lesions in viral and mycoplasma infections of the respiratory tract. Virchows Arch A Pathol Pathol Anat 1972;356: 259-73.

3 Green RJ, Zar HJ, Jeena PM, et al. South African guideline for the diagnosis, management and prevention of acute viral bronchiolitis in children. S Afr Med J 2010;100:320, 22-5.

4 Friedman JN, Rieder MJ, Walton JM, et al. Bronchiolitis: recommendations for diagnosis, monitoring and management of children one to 24 months of age. Paediatr Child Health 2014;19:485-98.

5 Ralston SL, Lieberthal AS, Meissner HC, et al. Clinical practice guideline: the diagnosis, management, and prevention of bronchiolitis. Pediatrics 2015;136:782.

6 National Institute for Health and Care Excellence. Bronchiolitis: diagnosis and management in children (Clinical guideline ng9), 2015.

7 Tapiainen T, Aittoniemi J, Immonen J, et al. Finnish guidelines for the treatment of laryngitis, wheezing bronchitis and bronchiolitis in children. Acta Paediatr 2016;105:44-9.

8 De Wilde S, Carey IM, Bremner SA, et al. A comparison of the recording of 30 common childhood conditions in the Doctor's Independent Network and General Practice Research Databases. Health Stat Q 2004;22:21-31.

9 Murray JC. The clinical burden of respiratory syncytial virus (RSV) bronchiolitis among infants in the United Kingdom (UK) [PhD]. London, UK: Imperial College, 2013.

10 Bueno Campana M, Calvo Rey C, Vazquez Alvarez $\mathrm{MC}$, et al. [Viral respiratory tract infections in the first six months of life]. An Pediatr (Barc) 2008;69: 400-5.

11 Kabir ML, Haq N, Hoque M, et al. Evaluation of hospitalized infants and young children with bronchiolitis - a multicentre study. Mymensingh Med J 2003;12:128-33.

12 Green CA, Yeates D, Goldacre A, et al. Admission to hospital for bronchiolitis in England: trends over five decades, geographical variation and association with perinatal characteristics and subsequent asthma. Arch Dis Child 2016;101:140-6.

13 Nasreen S, Luby SP, Brooks WA, et al. Population-based incidence of severe acute respiratory virus infections among children aged $<5$ years in rural Bangladesh, June-October 2010. PLOS ONE 2014;9: e89978.

14 Lukšić I, Kearns PK, Scott F, et al. Viral etiology of hospitalized acute lower respiratory infections in children under 5 years of age-a systematic review and meta-analysis. Croat Med J 2013;54:122-34.

15 Nair H, Nokes DJ, Gessner BD, et al. Global burden of acute lower respiratory infections due to respiratory syncytial virus in young children: a systematic review and meta-analysis. Lancet 2010;375:1545-55.

16 Modjarrad K, Giersing B, Kaslow DC, et al. WHO consultation on Respiratory Syncytial Virus Vaccine Development Report from a World Health Organization Meeting held on 23-24 March 2015 Vaccine 2016;34:190-7.

17 Mazur NI, Martinon-Torres F, Baraldi E, et al. Lower respiratory tract infection caused by respiratory syncytial virus: current management and new therapeutics. Lancet Respir Med 2015;3: 888-900. 